\title{
The Interaction Between Pubertal Timing and Peer Popularity for Boys and Girls: An Integration of Biological and Interpersonal Perspectives on Adolescent Depression
}

\author{
Hanneke A. Teunissen - Caroline B. Adelman • Mitchell J. Prinstein • \\ Renske Spijkerman • Evelien A. P. Poelen • Rutger C. M. E. Engels • Ron H. J. Scholte
}

Published online: 9 November 2010

(C) The Author(s) 2010. This article is published with open access at Springerlink.com

\begin{abstract}
The transition to adolescence marks a time of sharply increased vulnerability to the development of depression, particularly among girls. Past research has examined isolated risk factors from individual theoretical models (e.g., biological, interpersonal, and cognitive) of depression, but few have examined integrative models. This study investigated the conjoint effects of early pubertal timing and popularity in the longitudinal prediction of depressive symptoms. A total of 319 girls and 294 boys (ages 11-14) provided information on their pubertal status, depressive symptoms, and the social status (i.e., popularity) of their peers. Adolescents completed a second measure of depressive symptoms 11 months after the initial time point. Findings supported an integrated biological-interpersonal model in explaining the development of depressive symptoms during adolescence. Early pubertal development was associated with increase in depressive symptoms only when accompanied by low levels of popularity. High levels of popularity buffered the association between early pubertal development and later depressive symptoms. Unexpectedly, these results were significant both for girls and boys. Results are discussed in terms of dynamic systems theories.
\end{abstract}

Keywords Pubertal timing - Depressive symptoms . Peer status

\footnotetext{
H. A. Teunissen $(\triangle) \cdot$ R. Spijkerman $\cdot$ E. A. P. Poelen $\cdot$

R. C. M. E. Engels · R. H. J. Scholte

Behavioural Science Institute, Radboud University Nijmegen,

Post box 9102, 6500 HC Nijmegen, The Netherlands

e-mail: H.Teunissen@pwo.ru.nl

C. B. Adelman • M. J. Prinstein

Department of Psychology, University of North Carolina,

Chapel Hill, NC, USA
}

Dynamic systems perspectives of psychology suggest that behavior may be best understood through consideration of multiple interacting systems of development (Magnusson and Cairns 1996). Unfortunately, current literature too often focuses on individual risk factors, without regard for contextual, environmental, cultural, or maturational systems with which these risk factors may transact. Research regarding the unique risks for depressive symptoms among adolescent girls has been limited by this somewhat myopic perspective.

To date, separate risk factors models (e.g., cognitive, biological, interpersonal) have been proposed to understand the dramatic increase in depression prevalence among girls that occurs at the adolescent transition (Hankin and Abramson 2001). For example, cognitive models of depression generally have suggested that individuals' global attribution style regarding stressful life events may interact with the occurrence of actual stressors to confer heightened risk for depressive symptoms (e.g., Abramson et al. 1989, 1978; Gladstone and Kaslow 1995). Biological models of adolescent depression have suggested that pubertal changes in circulating hormones may be linked to increases in negative affect (Angold et al. 1999; Benjet and Hernández-Guzmán 2002; Crockett and Petersen 1987). In addition, atypical pubertal timing may be associated with the onset of depressive symptoms among girls (Brooks-Gunn 1988; Rierdan and Koff 1991; Stice et al. 2001). Interpersonal models suggest reciprocal associations between depressive symptoms and interpersonal rejection that lead to exacerbations of depressed affect over time. Despite the important advances in studies utilizing each of these discrete theoretical perspectives, few studies have examined integrative models in which interactions between these risk factors may be especially relevant for the development of depression. A developmental psycho- 
pathology approach allows for a theoretical conceptualization of how these factors evolve over maturational periods and interact in unique ways that confer risks for depressive symptoms, perhaps especially for girls.

Adolescence is marked by a unique constellation of changes in cultural, social, maturational, contextual, and biological systems (Hinshaw and Kranz 2009). Biologically, the adolescent transition is associated with puberty-related changes in circulating hormones, stress-reactivity processes, and body shape. Several studies have suggested that pubertal development is associated with changes in depressive symptoms (Angold et al. 1998; Benjet and HernándezGuzmán 2002; Ge et al. 2001). A study by Patton and colleagues (2008) showed that advancing pubertal stage predicted the onset of depressive symptoms, especially in girls, and that advancing pubertal stage more than redoubled the risk for continuing depressive symptoms for girls in mid- to late puberty. However, research has suggested that aberrations in pubertal timing may be especially relevant to depressive symptoms, particularly for girls (Ge et al. 1996; 2003; Rierdan and Koff 1991). Thus, some of the effects of pubertal development are specific to the interaction between biological factors and contextual factors, such as the comparative development of adolescents' peers (e.g. Caspi and Moffitt 1991; KaltialaHeino et al. 2003).

Specifically, adolescents who develop early as compared to their peers may experience altered trajectories of social development within the peer context. As compared to others, early-developing adolescents appear different from their age mates, and consequently are met with societal expectations that may be mismatched to their cognitive and emotional development (i.e., a stage-termination hypothesis; e.g. Silbereisen and Kracke 1997). Early pubertal development among girls is associated with disapproval among peers, evidenced by increasing social isolation, peer victimization, or negative social reputations among peers (Conley and Rudolph 2009; Craig et al. 2001; Nadeem and Graham 2005).

Research examining gender differences in depression suggests that this sort of biologically-prompted social stress may be an especially salient risk factor for depression among girls. As compared to younger children, and to adolescent boys, adolescent girls experiencing the pubertal transition have a heightened sensitivity to their body shape and to social comparisons with peers regarding the romantic appeal of their bodies (Carlson Jones 2001). Early maturing girls report high levels of body dissatisfaction, which can be explained by the experienced deviation not only from the cultural norm of slimness, but also from their same-aged peers (Alsaker 1995; Carlson Jones 2001; Dorn et al. 1988). With increased cognitive abilities in adolescence, girls are especially capable of making these social comparisons and abstracting a global sense of self from negative reactions among peers. Indeed, numerous studies have suggested that early-maturing girls are at especially high risk for depressive symptoms (Ge et al. 1996, 2003; Graber et al. 2003; Kaltiala-Heino et al. 2003; Rierdan and Koff 1991). Models specifically examining interactions between early pubertal development and interpersonal factors are rare, however.

Adolescence also is accompanied by dramatic changes in youths' interpersonal experiences, particularly among peers. As compared to younger children, adolescents spend increased time with peers, while experiencing more autonomy from parents (Savin-Williams and Berndt 1990). The adolescent task of identity formation becomes especially dependent on social comparisons and reflected appraisals with peers (Kroger 1996), rendering adolescents' level of peer status a highly salient and important determinant of adolescents' self-perceptions. This also may be especially relevant for adolescent girls. Research suggests that in adolescence, girls experience more interpersonal stress than do boys (Rudolph and Hammen 1999; Rudolph 2002; Rudolph et al. 2001), and these stressors increase with girls' age (Rose and Rudolph 2006). Relative to males, females are more likely to base their self-esteem on their interpersonal relationships (e.g., Chevron et al. 1978) and more likely to experience depressive symptoms following interpersonal stress (e.g., Rudolph and Hammen 1999).

The adolescent transition also is associated with unique adaptations in the type and meanings of peer reputations. Prior to adolescence, youths' reputations among peers may be characterized by the extent to which they are well-liked by others. Typically measured through the use of preference-based peer nominations (i.e., assessing who peers like the most and like the least), peer likeability or acceptance/rejection frequently has been studied as a predictor of psychological risk or protection. Children who are rejected by peers are at greater risk than others for later externalizing symptoms, school dropout, and health risk behaviors (Coie et al. 1995; Parker and Asher 1987). Acceptance by peers, in contrast, may buffer the effects of girls' childhood aggression on later externalizing outcomes (Prinstein and La Greca 2004).

Recent research has suggested that at the adolescent transition, a discrete measure of peer status emerges (i.e., "peer perceived popularity"1). As compared to peer acceptance/rejection, this reputation-based measure of peer status is based on nominations of peers who are "most-" and "least-popular", yielding a measure that suggests dominance, power, and access to resources among adoles-

\footnotetext{
"From now on, "peer-perceived popularity" will be referred to as "popularity".
} 
cents (Cillessen and Rose 2005; Parkhurst and Hopmeyer 1998). Popularity also is highly correlated with measures of physical attractiveness and idealized body shapes (Boyatzis et al. 1998; LaFontana and Cillessen 2002; Wang et al. 2006). Although preference- and reputation-based measures of peer status (i.e., likeability and popularity) are correlated quite strongly in childhood, these two measures of peer status differentiate as children mature (Cillessen and Mayeux 2004). By the adolescent transition, popularity is an important, discrete construct that is especially salient to adolescents as a measure of favorable regard among peers. Adolescents desire to achieve high levels of popularity has been linked to strong motivations for peer conformity (Cillessen and Rose 2005); accordingly low levels of popularity may be associated with poor self-regard and risk for depressive symptoms.

This study examined an interaction between pubertal timing and a reputation-based measure of peer status, as a longitudinal predictor of adolescents' depressive symptoms. It was anticipated that among early maturing adolescents, low levels of popularity would be associated with increasing levels of depressive symptoms over time; however, similar effects would not be revealed for on-time maturing adolescents, or for early maturing adolescents with high levels of popularity. It was further expected that this finding would be moderated by gender, such that the interaction between early pubertal development and popularity would be a significant longitudinal predictor of depressive symptoms for girls, but not for boys. This can be understood by the assumption that at least in Western cultures, adolescents' biological and interpersonal systems are interconnected (Lerner 1985) and their interaction should therefore be taken into account when predicting depressive symptoms. Some adolescent girls in Western society experience an internalization of cultural expectations of femininity. Bem (1974) suggests that Western culture promotes unique gender-specific expectations (e.g., strength and power among males; physical beauty and interpersonal skill among females). Negative experiences that are unrelated to gender stereotypes (i.e., threats to strength and power among females; threats to sensitivity and interpersonal skill among males) may not be strongly related to distress. However, experiences that are discordant with sex-typed expectations (e.g., for girls: violations of ideal body shape norms, social rejection) yield vulnerabilities for unfavorable self-evaluation, and perhaps depressive symptoms. The adolescent transition therefore represents a unique period for girls, during which changes in physical appearance and/or negative experiences in the interpersonal domain, may be especially likely to clash with cultural expectations for girls, and thus yield additional risk for depressive symptoms. Difficulties with both of these areas (i.e., early pubertal development altering body shape with respect to one's peers combined with low popularity) would be especially relevant to feminine cultural norms.

\section{Methods}

Participants included 613 youth (52\% female) enrolled in grades $6(35 \%), 7(29 \%)$ and $8(36 \%)$ at study outset. The ethnic composition of the sample included $86 \%$ White/ Caucasian, 4\% Asian-American, 2\% Latino/a-American, $1 \%$ African-American, and $6 \%$ mixed ethnicity students. Participants were enrolled in a single public middle school within a city of fairly homogeneous middle-class socioeconomic status. According to school records, $11 \%$ of children were eligible for free/reduced-price lunch.

At Time 1, all sixth through eighth grade students and their families received mailed and hand-distributed consent forms for study recruitment with strong encouragement and incentives (e.g., raffles) for consent form return. Consent forms were returned by $92 \%$ of families $(n=784)$; of these, $80 \%$ of parents gave consent for their child's participation ( $n=627 ; 74 \%$ of the total population). Students who were absent on one of the days of testing $(n=10)$ or refused to participate $(n=4)$, were excluded from analyses, yielding a final sample of 613 participants at Time 1. A total of 567 $(92 \%)$ of these participants were available for testing eleven months later (i.e., Time 2), when students were in grades seven through nine. Attrition was due to participants moving away from the area $(n=36)$, absenteeism $(n=7)$, and three participants who refused to continue participation. No significant differences were revealed for any of the constructs measured in this study between adolescents who participated at both time points and those who only participated at one time point. Because listwise deletion would unnecessarily omit valuable data, all analyses were conducted with all available data (see "Data Analyses" below). Analyses using only available data revealed an identical pattern of results.

At Time 1, measures of pubertal timing, depressive symptoms, and reputation-based peer status (i.e., popularity) were administered. Eleven months later, when students were in grades 7-9 (i.e., Time 2), participants completed a second measure of depressive symptoms.

\section{Measures}

Pubertal Timing The Pubertal Development Scale (Petersen et al. 1988) includes five items for boys (e.g., growth spurt, body hair, skin change, voice change, facial hair) and girls (e.g., growth spurt, body hair, skin change, breast growth, menarche) measuring gender-specific physical changes associated with maturation. Adolescents respond to each item using a four point Likert scale $(1=$ 
not started; $4=$ seems completed). As in past research (McBride et al. 2003), responses for girls' menarche were coded $(1=$ no; $4=$ yes $)$ to create a scale comparable to other items and a mean score across all five items was computed for both girls, $\alpha=0.75$, and boys, $\alpha=0.79$, with higher scores indicating more advanced pubertal development. Pubertal development scores were standardized within grade, within gender to obtain a measure of pubertal timing.

Popularity A sociometric peer nomination questionnaire was used to measure the peer status of participants. Students in this school were grouped in academic teams. Each team was about twice the size of a traditional academic classroom $(n s=30-75)$. An alphabetized list of all teammates was presented to each participant and they were asked to select an unlimited number of peers that were "most popular" and peers that were "least popular". The order of the names on this list was counterbalanced (A through $\mathrm{Z}$ or $\mathrm{Z}$ through $\mathrm{A}$ ) to control for possible effects of order on the selection of peers. For each participant the total number of nominations received was computed and standardized within each academic team. The difference between standardized "most popular" and "least popular" scores then was computed and re-standardized as a measure of social reputation, with higher scores indicating higher levels of popularity. Peer nominations are considered the most reliable and valid method to measure various dimensions of peer status and reputations in adolescence (Jiang and Cillessen 2005).

Depressive Symptoms The Children's Depression Inventory (CDI; Kovacs 1981, 1985) is a 27-item measure designed to assess depressive affect and symptoms. For each item, the participant is provided with three alternative statements and instructed to select the one that best describes their symptoms over the past 2 weeks (e.g., "I am sad once in a while, "I am sad many times," and "I am sad all the time"). Responses are coded on a scale of $0-2$, with higher scores indicating heightened depressive symptoms. The CDI is well-established as a reliable and valid assessment of depressive symptoms in children between the ages of 7 and 18 years (Smucker et al. 1986).

\section{Data Analyses}

Descriptive statistics first were conducted to examine the gender differences in the primary study variables, as well as intercorrelations. Hypotheses were examined with a multiple group (by gender) structural equation model using full information maximum likelihood as implemented in Amos version 16.0. Means and intercepts were estimated using full information direct maximum likelihood when data were missing.
Depressive symptoms at Time 1 and Time 2 were estimated as latent variables by creating three item parcels of the CDI. A parcel is a subset of items from the item pool of 27 items. Each parcel is the summed score on a subset of 9 items. To create parcels the underlying latent variable must be unidimensional. We examined the unidimensionality of the CDI using Confirmatory Factor Analysis (CFA). ${ }^{2}$ From these results we may infer that the latent variable 'depressive symptoms' can be interpreted as a unidimensional variable. For the construction of the three parcels we used the item-to-construct balance method as described in Little et al. (2002). A 1-factor solution at Time 1 was used to allocate items to one of the three parcels according to the magnitude of the factor loadings. In this way each parcel reflected the original factor structure with higher and lower factor loadings. Identical parcels were formed at T2. The alpha's of the parcels at T1 were $0.75,0.76$ and 0.75 , and at T2 $0.77,0.75,0.68$. In the model error terms of identical parcels over time were correlated (Finkel 1995). ${ }^{3}$

A multiple group (by gender) structural equation model was estimated to examine the primary hypotheses regarding a three-way interaction between pubertal timing, popularity, and gender. The model included an estimated autocorrelation between latent variables for depressive symptoms at Time 1 to Time 2. Paths were estimated between both exogenous predictors (i.e., pubertal timing, popularity) and Time 2 depressive symptoms, as well as between a product term between these two variables (pubertal timing $\mathrm{x}$ popularity) predicting Time 2 depressive symptoms. To examine hypotheses in a stringent manner, two additional variables were entered as predictors: age, and a product term between age and popularity. All Time 1 variables were allowed to covary. The error terms for the depressive symptoms parcels at Time 1 were allowed to correlate with the error terms for the corresponding depressive symptoms parcels at Time 2. Gender interactions were examined by

\footnotetext{
${ }^{2}$ Because the response scales of the 27 items consist of 3 categories $(0,1$, 2) and have very skewed distributions (i.e., a preponderance of zero's), we used Mplus (Muthén and Muthén 1998-2007) because this program has facilities to deal with this type of data. We used the WLSMestimator ( $\mathrm{M}=$ Mean-adjusted chi-square test). A CFA with one factor showed a good fit at $\mathrm{T} 1\left(\chi^{2}(324)=711.10, \mathrm{CFI}=0.971\right.$ and RMSEA $=$ $0.061)$ and $\mathrm{T} 2\left(\chi^{2}(324)=719.84, \mathrm{CFI}=0.958\right.$ and $\left.\mathrm{RMSEA}=0.065\right)$. The factor loadings at $\mathrm{T} 1$ varied between 0.37 and 0.91 (mean factor loading was $0.67, S D=0.13$ ) and at $\mathrm{T} 2$ between 0.37 and 0.86 (mean factor loading was $0.66, S D=0.13$ ). At T1 Cronbach's alpha $=0.90$, with corrected item-total correlations varying from $0.28-0.65(M=0.48, S D=$ 0.10 ). At $\mathrm{T} 2 \mathrm{alpha}=0.89$, corrected item-total correlations varying from $0.27-0.62(M=0.47, S D=0.09)$.

${ }^{3}$ All analyses also were conducted using a total depressive symptoms score at Times 1 and 2 as an observed variable in hierarchical multiple regressions. These analyses therefore included observed variables for all primary study constructs (i.e., pubertal timing, popularity, and age). The findings using this more simplified procedure were identical to those reported below.
} 
comparing models with paths either fixed or free to vary between groups, and the significance of chi-squared difference tests between nested models was used to examine statistically significant gender differences in the magnitude of estimated paths. Unless otherwise noted, all parameter estimates were allowed to vary freely by gender (see Fig. 1).

\section{Results}

Means and standard deviations for all variables included in this study are presented in Table 1. As would be anticipated,
T-tests indicated that girls reported higher levels of depression at Time 2 than boys. Pearson correlations between all variables are presented in Table 2. Pubertal timing was positively related to depressive symptoms at Time 1 and Time 2 (for girls only). Low levels of popularity were associated with high levels of depressive symptoms at Time 1 (for boys and girls) and Time 2 (for girls only).

Results suggested that an initial model with all paths, factor weights, covariances, and error terms freely varying across gender was a satisfactory fit to the data, $\chi^{2}(104)=$ 85.99, $p<0.001 ; \chi^{2} / \mathrm{df}=1.72 ; \mathrm{CFI}=0.99 ; \mathrm{RMSEA}=0.03$. Paths, covariances, and error terms systematically were constrained across gender to examine improvement in
Fig. 1 Structural equation model examining popularity as a moderator of the longitudinal association between pubertal timing and depressive symptoms, while controlling for age effects

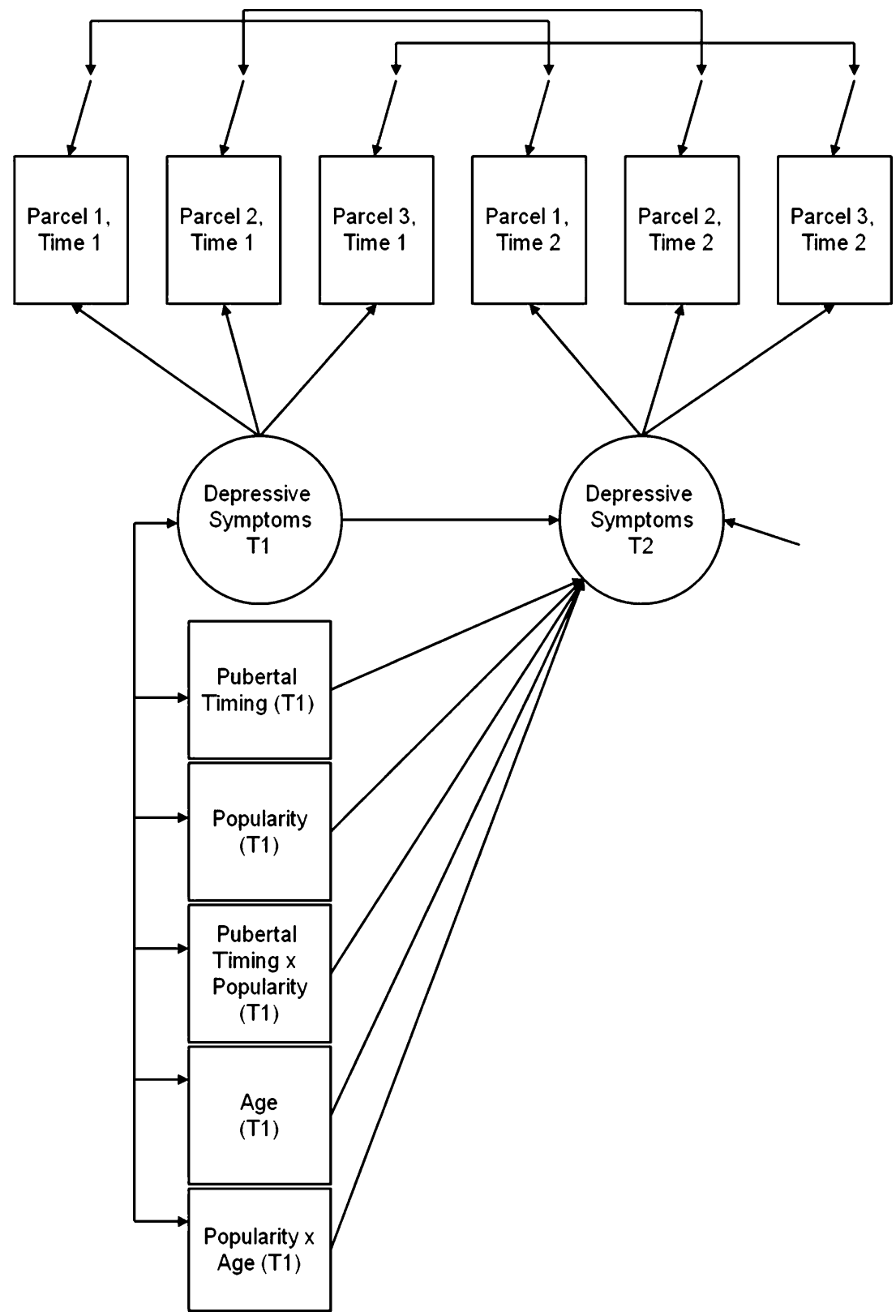


Table 1 Means (and standard deviations) for primary variables at Time 1 and Time 2

${ }^{*} p<0.05 ; * * p<0.0001$

\begin{tabular}{lccc}
\hline & \multicolumn{1}{l}{ Boys } & Girls & $t(613)$ \\
\hline Time 1 & & & \\
Depressive Symptoms & $0.27(0.24)$ & $0.30(0.27)$ & -1.13 \\
Pubertal Development (unstandardized) & $2.30(0.63)$ & $2.77(0.73)$ & $-8.91^{* *}$ \\
Popularity & $-0.03(1.03)$ & $0.09(0.93)$ & -1.46 \\
Age (in months) & $151.57(11.64)$ & $150.78(10.84)$ & 0.88 \\
Time 2 & & $0.25(0.25)$ & $-2.02^{*}$ \\
Depressive Symptoms & $0.21(0.20)$ & & \\
\hline
\end{tabular}

model fit. For all but five estimated parameters, estimates could be fixed across gender without significant detriment to model fit. Two of these parameters represented gender differences in covariances: (1) covariance between the two product terms in model: 0.61 for girls, NS; 3.64 for boys, $p<$ $0.001 ; \Delta \chi^{2}=13.82, p<0.001$; (2) covariance between pubertal timing and depressive symptoms: 0.35 for girls, $p<0.01$; -0.10 for boys, NS; $\left.\Delta \chi^{2}=7.46, p<.01\right)$. Two parameters represented differences in factor weights (1) parcel 2 at Time 2: 1.03 for girls, $p<0.001 ; 1.27$ for boys, $p<0.001 ; \Delta \chi^{2}=$ 5.23, $p<0.05$; (2) parcel 3 at Time 2: 0.95 for girls, $p<0.001$; 1.36 for boys, $p<0.001 ; \Delta \chi^{2}=14.04, p<0.001$. Last, the autocorrelation of depressive symptoms over time was moderated by gender: 0.82 for girls, $p<0.001$; 0.51 for boys, $p<0.001 ; \Delta \chi^{2}=15.11, p<0.001$. Thus, these estimates remained freely varying.

All path coefficients are presented in Table 3. The autocorrelation path between depressive symptoms at both time points was statistically significant for boys and girls. After accounting for this association, no significant interaction between age and popularity was revealed in the prediction of depressive symptoms either for boys or girls. The results suggested that the interaction term (pubertal timing x popularity) was associated significantly with Time 2 depressive symptoms (see Table 3 ). However, contrary to hypotheses, a chi-square difference test suggested no significant detriment to model fit when constraining this path across gender as compared to when allowing this path to vary freely, $\chi^{2}(1)=2.44, p=0.12$, suggesting that gender did not further moderate the pubertal timing $\mathrm{x}$ popularity interaction. The final model was a good fit to the data, $\chi^{2}$ $(72)=104.79, p<0.01 ; \chi^{2} / \mathrm{df}=1.46 ; \mathrm{CFI}=0.99$; $\mathrm{RMSEA}=$ 0.03 (90\% CI 0.01 to 0.04$)$

To examine the interaction between pubertal timing and popularity, a reduced model next was estimated including only prior levels of depressive symptoms, the main effects of hypothesized predictors, and interaction term (i.e., removing age and the interaction between age and popularity as predictors to ensure that findings were not due to suppression effects; Holmbeck 2002). This reduced model was a good fit to the data, $\chi^{2}(46)=81.72, p<0.01 ; \chi^{2} / \mathrm{df}=1.78 ; \mathrm{CFI}=0.98$; $\mathrm{RMSEA}=0.03$. The interaction between pubertal timing and popularity as a longitudinal predictor of depressive symptoms remained significant, $b=-0.14, p<0.05$. Results of simple slope analyses were conducted for adolescents scoring low (-1 SD), average, or high (+1 SD) in popularity. For adolescents with low levels of popularity, advanced pubertal timing compared to peers (i.e., early pubertal timing) was associated with high levels of depressive symptoms at Time 2, slope $=0.18, t=2.11, p<0.05$. For

Table 2 Bivariate associations among primary variables

\begin{tabular}{|c|c|c|c|c|c|}
\hline & \multicolumn{4}{|l|}{ Time 1} & \multirow{2}{*}{$\begin{array}{l}\text { Time } 2 \\
\text { Depressive symptoms }\end{array}$} \\
\hline & Depressive symptoms & Pubertal timing & Popularity & Age & \\
\hline \multicolumn{6}{|l|}{ Time 1} \\
\hline Depressive symptoms & & 0.02 & $-0.19 * *$ & 0.07 & $0.60 * * *$ \\
\hline Pubertal Timing & $0.12 *$ & & 0.09 & $0.13 *$ & 0.02 \\
\hline Popularity & $-0.20 * *$ & 0.03 & & 0.08 & -0.10 \\
\hline Age & 0.00 & 0.10 & 0.04 & & $0.14^{*}$ \\
\hline \multicolumn{6}{|l|}{ Time 2} \\
\hline Depressive symptoms & $0.75^{* * *}$ & $0.16^{* *}$ & $-0.19 * *$ & $0.05 *$ & \\
\hline
\end{tabular}

Correlations for males appear above the diagonal; correlations for females appear below the diagonal.

${ }^{*} p<0.05 ; * * p<0.01 ; * * * p<0.001$ 
Table 3 Unstandardized path coefficients, standard errors, and standardized path coefficients for structural equation model examining longitudinal associations between pubertal timing, popularity, age, and depressive symptoms at Time 1 with depressive symptoms at Time 2, for boys and girls

Unstandardized (SE) Standardized

\begin{tabular}{llll} 
Time 1 & & & \\
Depressive symptoms & a & & \\
Pubertal timing & 0.03 & $(0.06)$ & 0.01 \\
Popularity & 0.01 & $(0.06)$ & 0.00 \\
Age & 0.01 & $(0.01)$ & $0.07^{*}$ \\
Age $\times$ popularity & 0.01 & $(0.01)$ & 0.03 \\
Pubertal timing $\times$ popularity & -0.29 & $(0.11)$ & $-0.11^{*}$ \\
\hline
\end{tabular}

${ }^{a}$ This path weight was moderated by gender, Girls: Unstandardized= $0.82(0.05)$, Standardized $=0.81^{* *}$; Boys: Unstandardized $=0.51(0.05)$, Standardized $=0.67 * *$

${ }^{*} p<0.01 ; * * p<0.001$

adolescents average or high in popularity, the association between pubertal timing was not associated significantly with Time 2 depressive symptoms, slopes $=0.04$ and -0.09 , $t_{s}=0.81$ and $-1.14, N S$, respectively.

\section{Discussion}

This study examined popularity as a potential moderator of the relationship between early pubertal timing and the development of depressive symptoms over a one year interval. It was expected that this effect would be revealed only for girls. Results revealed a significant moderating effect of popularity, however, surprisingly, significant effects were revealed both for boys and girls. Specifically, the positive relationship between early pubertal timing and increases in depressive symptoms was revealed for adolescents with a low social reputation, but not among adolescents with a high social reputation. These results suggest that a high social reputation may protect early maturing adolescents from the development of depressive symptoms over time, while a low social reputation may be a risk factor for the development of depressive symptoms.

The findings supported an integrated biologicalinterpersonal model in examining the development of depressive symptoms during adolescence. The interaction between pubertal timing and social reputation in predicting the change in depressive symptoms may explain why studies that have focused solely on the association between early pubertal timing and depressive symptoms, or on the relationship between social reputation and depressive symptoms, have yielded mixed results (e.g. Canals et al. 1995; Ge et al. 2003; La Greca and Harrison 2005; Prinstein and La Greca 2002; Stroud and Davila 2008).
The additional consideration of peer status as a moderator is consistent with biopsychosocial models, which remind us to consider transactions that occur between social, biological and psychological systems in understanding the development of psychopathology. It appears that the inclusion of peer status as a moderator provides a more complete picture of the relationship between early pubertal development and the development of depressive symptoms over time.

The finding that interpersonal factors interact with pubertal timing in predicting depressive symptoms is consistent with a study by Conley and Rudolph (2009), which revealed that early pubertal timing was related to depressive symptoms in adolescents who experienced high levels of peer stress, but not in adolescents who experienced low levels of peer stress. Examples of peer stress included fights with peers or being teased by peers, social exclusion, and low support or poor quality of friendships. The authors suggested that early pubertal timing is a diathesis for depressive symptoms that is activated when it occurs in a stressful peer context. This model offers one possible interpretation of the current study's findings: popularity may reduce the incidence of certain interpersonal stressors associated with early maturation (i.e., reduce the likelihood of peer victimization), and thus may serve as a protective factor against the development of depressive symptoms. Further studies assessing the incidence of peer victimization and other social stressors among popular versus unpopular early developing adolescents could determine whether popularity does, in fact, buffer against depression by decreasing the incidence of social stressors. Such a finding would provide evidence that the moderational effect of peer status may be a proxy for increased exposure to social stressors, which interact with the biological (i.e., hormonal) and social-cognitive (i.e., selfappraisal) vulnerabilities posed by early development to predict the onset of depressive symptoms.

Notably, however, Conley and Rudolph's (2009) results were significant for girls, and not for boys. Although the data in this study trended in a similar direction, the role of gender in further moderating hypothesized effects did not reach significance. In addition, absent substantial data confirming the measurement and scalar invariance of the CDI across gender, it is difficult to make strong interpretations regarding gender effects conclusively (see Carle et al. 2008). It may be that among males, early pubertal timing combined with low peer popularity also presents risks for depressive symptoms over time. It is likely that early pubertal timing may become a source of ridicule or ostracism among boys who already are unpopular, but perhaps a source of status and esteem among boys who enjoy high levels of popularity. This unexpected result deserves further examination in future research. 
This study represents a fruitful extension of past research. The finding that the relationship between early pubertal timing and depressive symptoms is moderated by popularity is a step forward in understanding both the inconsistent findings of past research in this domain, and the uniqueness of the adolescent period in the development of depression. Furthermore, the use of a longitudinal, prospective design allowed for examination of the temporal relationship between these variables.

Despite these strengths, several limitations of the current study highlight the somewhat tentative nature of these results. First, results of Conley and Rudolph's (2009) study discussed above serve as a reminder that the construct of peer status may serve as a proxy for level of social stress or other related variables. Indeed, several interpersonal variables (e.g., social stress, peer-perceived attractiveness, friendship quality, etc.) correlate highly with popularity, and cannot be meaningfully examined as orthogonal moderators of the relationship between early pubertal development and depression. Other variables that correlate with popularity could explain the difference in depressive symptoms between popular and unpopular early maturing youth. Socioeconomic status, for example, is positively correlated with membership in the popular group (Brown et al. 1993; Eder 1985). In the present study however, all participants had similar SES, so in our sample this factor could not explain the relationship between popularity and the change in depressive symptoms in early maturing adolescents.

Another important factor that might explain the difference in depressive symptoms among popular and unpopular early maturing adolescents is social connectedness. Adolescents with high social connectedness feel close with peers, easily identify with others and participate in social activities (Lee et al. 2001). Popularity is associated with social connectedness, while unpopularity is related to social withdrawal and a lack of skills to fit in with peers (LaFontana and Cillessen 2002). Other findings indicate a negative relationship between social connectedness and depressive symptoms, suggesting that adolescents who are socially less well connected are more likely to experience depressive symptoms (Lee et al. 2001). This could suggest that low levels of social connectedness in unpopular adolescents are related to high levels of depressive symptoms. Social connectedness could then be the underlying factor that accounts for the difference in depressive symptom scores between popular and unpopular early maturing adolescents. Further research is needed to determine mechanisms by which early puberty confers risk for the development of depressive symptoms among adolescents. While the current study provides valuable information about one potential moderator of this relationship, interpretations regarding the process (i.e., how popularity may buffer against the development of depressive symptoms for early developing adolescents) remain theoretical.

Another limitation is the use of an ethnically homogenous, primarily Caucasian sample. Some research suggests that European American adolescents, but not African American adolescents, experience more depressive symptoms following early maturation (Michael and Eccles 2003). This difference might be explained by the finding that African and African-American women, in particular, report a preference for a body type and size that is more consistent with a post-pubescent body (i.e., larger and curvier), whereas Caucasian women report a preference for a thinner body type (Cogan et al. 1996; Furnham and Baguma 1994; Rucker and Cash 1992). Consistent with this idea, Siegel and colleagues (1999) found that African American girls, compared to Caucasian and Latino girls, are unique in their level of body satisfaction during adolescence. However, more recent research has found a positive relationship between early pubertal timing and depressive symptoms in African American girls (Ge et al. 2003). The mechanisms underlying this association are not well understood, and warrant further investigation.

Studies further examining the role of ethnicity in the association between pubertal timing and depressive symptoms should note that ethnicity should be considered in the context of the overall community in which adolescents live. Note that African-American adolescents develop earlier than Caucasian adolescents (Herman-Giddens et al. 1997). In a context predominantly comprised of Caucasian adolescents, African American adolescents are more likely to be characterized as early maturers, while they might be on-time compared to their African-American agemates. It may be important for research to consider pubertal timing with respect to same-ethnicity peers (Ge et al. 2003).

An additional limitation in this study pertains to the use of self-report measures to assess pubertal timing. Although the PDS is a widely used scale to measure pubertal development, it assesses perceived pubertal maturation instead of objective pubertal maturation. Adding more objective measures of pubertal maturation (e.g., physician ratings) would strengthen the reliability of our findings (Dorn et al. 2003). Conversely, the use of peer-reported measures to assess popularity offers the possibility that different peers (e.g., males vs. females) use different standards to determine who is popular. This issue also leads to invariance within the measurement of key constructs.

Despite these limitations, the present study provides insight into the relationship between early pubertal timing and the development of depressive symptoms. The results indicate that early pubertal timing does not directly predict depressive symptoms among adolescents. Instead, individual differences in popularity appear to affect the nature of 
this relationship. These findings indicate that the interaction between individual development and the peer context may be important for understanding the relationship between pubertal timing and depressive symptoms. Future studies are likely to benefit from a similar biopsychosocial perspective in understanding the complex interactions between various systems in determining typical versus atypical development.

The findings of the current study may have important implications for prevention of depressive symptoms. Our results provide a more complete profile of adolescents who may be at risk for the development of depressive symptoms. Interventions that target early maturing, unpopular adolescents therefore may serve to reduce the prevalence of depressive symptoms among youth. Evidence that social skills and social connectedness are related to both social status and depression (LaFontana and Cillessen 2002; Lee et al. 2001) suggests that interventions that focus on these constructs might help to reduce risk for depression among adolescents. Along these same lines, focusing on resiliency factors and resources for adolescents who are at risk may be beneficial. Examples of resiliency factors may include social skills and participation in extracurricular activities. The introduction of these and other resiliency factors through targeted interventions might aid in disconnecting the trajectory from risk factors to the development of depressive symptoms (Fergus and Zimmerman 2005).

Furthermore, psychoeducation also could be a crucial element in the prevention of depressive symptoms. Social comparisons play an important role during pubertal maturation. Early maturing adolescents may perceive themselves as deviant from their peers, and this perception of abnormality may negatively affect well-being. Psychoeducation aimed at informing adolescents about pubertal maturation, and the individual differences in its timing, might result in more personal and social acceptance of variations in puberty (Conley and Rudolph 2009). Psychoeducation also could help to prepare adolescents for the transition to puberty, and might even serve to reduce the accompanying psychosocial difficulties by normalizing the physical and social changes associated with pubertal development.

The current study has provided a clearer picture of the relationship between pubertal development, peer status, and the development of depressive symptoms over time. It appears that early pubertal timing and low social status converge to create a high-risk context for the development of depressive symptoms among adolescents. Treatment strategies that seek to alter this trajectory may benefit from strategies that both increase peer status, and decrease the psychosocial impact of early maturation. Several such strategies, suggested above, may represent complementary approaches in the reduction of depressive symptoms.
Open Access This article is distributed under the terms of the Creative Commons Attribution Noncommercial License which permits any noncommercial use, distribution, and reproduction in any medium, provided the original author(s) and source are credited.

\section{References}

Abramson, L. Y., Seligman, M. E. P., \& Teasdale, J. D. (1978). Learned helplessness in humans: critique and reformulation. Journal of Abnormal Psychology, 87, 49-74.

Abramson, L. Y., Metalsky, G. I., \& Alloy, L. B. (1989). Hopelessness depression: a theory based subtype of depression. Psychological Review, 96, 358-372.

Alsaker, F. D. (1995). Is puberty a critical period for socialization? Journal of Adolescence, 18, 427-444.

Angold, A., Costello, E. J., \& Worthman, C. M. (1998). Puberty and depression: the roles of age, pubertal status and pubertal timing. Psychological Medicine, 28, 51-61.

Angold, A., Costello, E. J., Erkanli, A., \& Worthman, C. M. (1999). Pubertal changes in hormone levels and depression in girls. Psychological Medicine, 29, 1043-1053.

Bem, S. L. (1974). The measurement of psychological androgyny. Journal of Consulting and Clinical Psychology, 42, 155-162.

Benjet, C., \& Hernández-Guzmán, L. (2002). A short-term longitudinal study of pubertal change, gender, and psychological wellbeing of Mexican early adolescents. Journal of Youth and Adolescence, 31, 429-442.

Boyatzis, C. J., Baloff, P., \& Durieux, C. (1998). Effects of perceived attractiveness and academic success on early adolescent peer popularity. The Journal of Genetic Psychology, 159, 337-344.

Brooks-Gunn, J. (1988). Antecedents and consequences of variations in girls' maturational timing. Journal of Adolescent Health Care, 9, 365-373.

Brown, B. B., Mounts, N., Lamborn, S. D., \& Steinberg, L. (1993). Parenting practices and peer group affiliation in adolescence. Child Development, 64, 467-482.

Canals, J., Marti-Henneberg, C., Fernández-Ballart, J., \& Domènech, E. (1995). A longitudinal study of depression in an urban Spanish pubertal population. European Child \& Adolescent Psychiatry, 4, $102-111$

Carle, A. C., Millsap, R. E., \& Cole, D. A. (2008). Measurement bias across gender on the Children's Depression Inventory: evidence for invariance from two latent variable models. Educational and Psychological Measurement, 68, 281-303.

Carlson Jones, D. (2001). Social comparison and body image: attractiveness comparisons to models and peers among adolescent girls and boys. Sex Roles, 45, 645-664.

Caspi, A., \& Moffitt, T. E. (1991). Individual differences are accentuated during periods of social change: the sample case of girls at puberty. Journal of Personality and Social Psychology, 61, 157-168.

Chevron, E. S., Quinlan, D. M., \& Blatt, S. J. (1978). Sex roles and gender differences in the experience of depression. Journal of Abnormal Psychology, 87, 680-683.

Cillessen, A. H. N., \& Mayeux, L. (2004). From censure to reinforcement: developmental changes in the association between aggression and social status. Child Development, 75, $147-163$.

Cillessen, A. H. N., \& Rose, A. J. (2005). Understanding popularity in the peer system. Current Directions in Psychological Science, 14, $102-105$.

Cogan, J. C., Bhalla, S. K., Sefa-Dedeh, A., \& Rothblum, E. D. (1996). A comparison study of United States and African 
students on perceptions of obesity and thinness. Journal of Cross-cultural Psychology, 27, 98-113.

Coie, J. D., Terry, R., Zakriski, A., \& Lochman, J. E. (1995). Early adolescent social influences on delinquent behavior. In J. McCord (Ed.), Coercion and punishment in long-term perspective (pp. 229244). New York: Cambridge University Press.

Conley, C. S., \& Rudolph, K. D. (2009). The emerging sex difference in adolescent depression: interacting contributions of puberty and peer stress. Development and Psychopathology, 21, 593-620.

Craig, W. M., Pepler, D., Connolly, J., \& Henderson, K. (2001). Developmental context of peer harassment in early adolescence: the role of puberty and the peer group. In J. Juvonen \& S. Graham (Eds.), Peer harassment in school (pp. 242-262). New York: The Guilford Press.

Crockett, L. J., \& Petersen, A. C. (1987). Pubertal status and psychosocial development: findings from the early adolescence study. In R. M. Lerner \& T. T. Foch (Eds.), Biologicalpsychosocial interactions in early adolescence (pp. 173-188). New Jersey: Lawrence Erlbaum Associates.

Dorn, L. D., Crockett, L. J., \& Petersen, A. C. (1988). The relations of pubertal status to intrapersonal changes in young adolescents. Journal of Early Adolescence, 8, 405-419.

Dorn, L. D., Susman, E. J., \& Ponirakis, A. (2003). Pubertal timing and adolescent adjustment and behavior: conclusions vary by rater. Journal of Youth and Adolescence, 32, 157-167.

Eder, D. (1985). The cycle of popularity: interpersonal relations among female adolescents. Sociology of Education, 58, 154165.

Fergus, S., \& Zimmerman, M. A. (2005). Adolescent resilience: a framework for understanding healthy development in the face of risk. Annual Review of Public Health, 26, 399-419.

Finkel, S. E. (1995). Causal analysis with panel data, Vol. 105. Thousand Oaks: SAGE.

Furnham, A., \& Baguma, P. (1994). Cross-cultural differences in the evaluation of male and female body shapes. The International Journal of Eating Disorders, 15, 81-89.

Ge, X., Conger, R. D., \& Elder, G. H., Jr. (1996). Coming of age too early: pubertal influences on girls' vulnerability to psychological distress. Child Development, 67, 3386-3400.

Ge, X., Elder, G. H., Jr., Regnerus, M., \& Cox, C. (2001). Pubertal transitions, perceptions of being overweight, and adolescents' psychological maladjustment: gender and ethnic differences. Social Psychology Quarterly, 64, 363-375.

Ge, X., Kim, I. J., Brody, G. H., Conger, R. D., Simons, R. L., Gibbons, F. X., et al. (2003). It's about timing and change: pubertal transition effects on symptoms of major depression among African American youths. Developmental Psychology, 39, 430-439.

Gladstone, T. R. G., \& Kaslow, N. J. (1995). Depression and attributions in children and adolescents: a meta-analytic review. Journal of Abnormal Child Psychology, 23, 597-606.

Graber, J. A., Seeley, J. R., Brooks-Gunn, J., \& Lewinsohn, P. M. (2003). Is pubertal timing associated with psychopathology in young adulthood? Journal of the American Academy of Child and Adolescent Psychiatry, 43, 718-726.

Hankin, B. L., \& Abramson, L. Y. (2001). Development of gender differences in depression: an elaborated cognitive vulnerabilitytransactional stress theory. Psychological Bulletin, 127, 773796.

Herman-Giddens, M. E., Slora, E. J., Wasserman, R. C., Bourdony, C. J., Bhapkar, M. V., Koch, G. G., et al. (1997). Secondary sexual characteristics and menses in young girls seen in office practice: a study from the pediatric research in office settings network. Pediatrics, 99, 505-512.

Hinshaw, S. P., \& Kranz, R. (2009). The triple bind: saving our teenage girls from today's pressures. New York: Ballantine.
Holmbeck, G. N. (2002). Post-hoc probing of significant moderational and mediational effects in studies of pediatric populations. Journal of Pediatric Psychology, 27, 87-96.

Jiang, X. L., \& Cillessen, A. H. N. (2005). Stability of continuous measures of sociometric status: a meta-analysis. Developmental Review, 25, 1-25.

Kaltiala-Heino, R., Kosunen, E., \& Rimpelä, M. (2003). Pubertal timing, sexual behaviour and self-reported depression in middle adolescence. Journal of Adolescence, 26, 531-545.

Kovacs, M. (1981). Rating scales to assess depression in school-aged children. Acta Paedopsychiatrica, 46, 305-315.

Kovacs, M. (1985). The Children's Depression Inventory. Psychopharmacology Bulletin, 21, 995-998.

Kroger, J. (1996). Identity in adolescence: the balance between self and other. London: Routledge.

LaFontana, K. M., \& Cillessen, A. H. N. (2002). Children's perceptions of popular and unpopular peers: a multimethod assessment. Developmental Psychology, 38, 635-647.

La Greca, A. M., \& Harrison, H. M. (2005). Adolescent peer relations, friendships and romantic relationships: do they predict social anxiety and depression? Journal of Clinical Child and Adolescent Psychology, 34, 49-61.

Lee, R. M., Draper, M., \& Lee, S. (2001). Social connectedness, dysfunctional interpersonal behaviors, and psychological distress: testing a mediator model. Journal of Counseling Psychology, 48, 310-318.

Lerner, R. M. (1985). Adolescent maturational changes and psychosocial development: a dynamic interactional perspective. Journal of Youth and Adolescence, 14, 355-372.

Little, T. D., Cunningham, W. A., Shahar, G., \& Widaman, K. F. (2002). To parcel or not to parcel: exploring the question, weighing the merits. Structural Equation Modeling: A Multidisciplinary Journal, 9, 151-173.

Magnusson, D., \& Cairns, R.B. (1996). Developmental science: toward a unified framework. In R.B. Cairns, G.H. Elder, Jr., E.J. Costello, \& A. McGuire (Eds.), Developmental Science (pp.7-30). New York: Cambridge University Press.

McBride, C. K., Paikoff, R. L., \& Holmbeck, G. N. (2003). Individual and familial influences on the onset of sexual intercourse among urban African American adolescents. Journal of Consulting and Clinical Psychology, 71, 159-167.

Michael, A., \& Eccles, J. S. (2003). When coming of age means coming undone: links between puberty and psychosocial adjustment among European American and African American girls. In C. Hayward (Ed.), Gender differences at puberty (pp. 277-303). New York: Cambridge University Press.

Muthén, L. K., \& Muthén, B. O. (1998-2007). Mplus user's guide, 5th edn. Los Angeles.

Nadeem, E., \& Graham, S. (2005). Early puberty, peer victimization, and internalizing symptoms in ethnic minority adolescents. Journal of Early Adolescence, 25, 197-222.

Parker, J. G., \& Asher, S. R. (1987). Peer relations and later personal adjustment: are low-accepted children at risk? Psychological Bulletin, 102, 357-389.

Parkhurst, J. T., \& Hopmeyer, A. (1998). Sociometric popularity and peer-perceived popularity: two distinct dimensions of peer status. Journal of Early Adolescence, 18, 125-144.

Patton, G. C., Olsson, C., Bond, L., Toumbourou, J. W., Carlin, J. B., Hemphill, S. A., et al. (2008). Predicting female depression across puberty: a two-nation longitudinal study. Journal of the American Academy of Child and Adolescent Psychiatry, 47, $1424-1432$.

Petersen, A. C., Crockett, L., Richards, M., \& Boxer, A. (1988). A self-report measure of pubertal status: reliability, validity, and initial norms. Journal of Youth and Adolescence, 17, $117-133$. 
Prinstein, M. J., \& La Greca, A. M. (2002). Peer crowd affiliation and internalizing distress in childhood and adolescence: a longitudinal follow-back study. Journal of Research on Adolescence, 12, 325-351.

Prinstein, M. J., \& La Greca, A. M. (2004). Childhood peer rejection and aggression as predictors of adolescent girls' externalizing and health risk behaviors: a 6-year longitudinal study. Journal of Consulting and Clinical Psychology, 72, 103-112.

Rierdan, J., \& Koff, E. (1991). Depressive symptomatology among very early maturing girls. Journal of Youth and Adolescence, 20, 415-425.

Rose, A. J., \& Rudolph, K. D. (2006). A review of sex differences in peer relationship processes: potential trade-offs for the emotional and behavioral development of girls and boys. Psychological Bulletin, 132, 98-131.

Rucker, C. E., \& Cash, T. F. (1992). Body images, body-size perceptions and eating behaviors among African-American and White college women. The International Journal of Eating Disorders, 12, 291-299.

Rudolph, K. D. (2002). Gender differences in emotional responses to interpersonal stress during adolescence. The Journal of Adolescent Health, 30, 3-13.

Rudolph, K. D., \& Hammen, C. (1999). Age and gender as determinants of stress exposure, generation, and reactions in youngsters: a transactional perspective. Child Development, 70, 660-677.

Rudolph, K. D., Lambert, S. F., Clark, A. G., \& Kurlakowsky, K. D. (2001). Negotiating the transition to middle school: the role of self-regulatory processes. Child Development, 72, 929-946.
Savin-Williams, R. C., \& Berndt, T. J. (1990). Friendship and peer relations. In S. S. Feldman \& G. R. Elliott (Eds.), At the threshold: The developing adolescent (pp. 277-307). Cambridge: Harvard University Press.

Siegel, J. M., Yancey, A. K., Aneshensel, C. S., \& Schuler, R. (1999). Body image, perceived pubertal timing, and adolescent mental health. The Journal of Adolescent Health, 25, 155-165.

Silbereisen, R. K., \& Kracke, B. (1997). Self-reported maturational timing and adaptation in adolescence. In J. Schulenberg, J. L. Maggs, \& K. Hurrelmann (Eds.), Health risks and developmental transitions during adolescence (pp. 85-109). Cambridge: Cambridge University Press.

Smucker, M. R., Craighead, W. E., Craighead, L. W., \& Green, B. J. (1986). Normative and reliability data for the Children's Depression Inventory. Journal of Abnormal Child Psychology, 14, 25-39.

Stice, E., Presnell, K., \& Bearman, S. K. (2001). Relation of early menarche to depression, eating disorders, substance abuse, and comorbid psychopathology among adolescent girls. Developmental Psychology, 37, 608-619.

Stroud, C. B., \& Davila, J. (2008). Pubertal timing and depressive symptoms in early adolescents: the roles of romantic competence and romantic experiences. Journal of Youth and Adolescence, 37, 953-966.

Wang, S. S., Houshyar, S., \& Prinstein, M. J. (2006). Adolescent girls' and boys' weight-related health behaviors and cognitions: associations with reputation- and preference-based peer status. Health Psychology, 25, 658-663. 